\title{
Quantum Effects on the Rayleigh-Taylor Instability of Viscoelastic Plasma Model through a Porous Medium
}

\author{
Gamal A. Hoshoudy ${ }^{1,2}$ \\ ${ }^{1}$ Department of Applied Mathematics, Faculty of Science, South Valley University, Kena, Egypt \\ ${ }^{2}$ Faculty of Girls Education at Muhayel, King Khalid University, Muhayel, Kingdom of Saudi Arabia \\ E-mail: g_hoshoudy@yahoo.com \\ Received June 8, 2011; revised July 13, 2011; accepted July 29, 2011
}

\begin{abstract}
The stability of stratified of incompressible, viscoelastic plasma through a porous medium in the presence of the quantum mechanism is considered. The dispersion relation is obtained using the normal mode technique. The behavior of growth rate with respect to the quantum effect, strain retardation time and stress relaxation time are examined in the presence of porosity of the porous medium, the medium permeability, kinematic viscosity. It is shown that, the presence of quantum term stabilizes a certain wave number band, whereas the system is unstable for all wave numbers in the absence of quantum term. The considered parameters beside the quantum term will bring about more stability on the considered system.
\end{abstract}

Keywords: Rayleigh-Taylor Instability, Viscoelastic, Quantum Plasm

\section{Introduction}

The Rayleigh-Taylor instability (RTI) is an important hydrodynamic effect that arises when a heavy fluid is accelerated into a lighter one. Similar to pouring water into oil, the heavier fluid, once perturbed, streams to the bottom, pushing the light fluid aside. This notion for a fluid in a gravitational field was first discovered by Rayleigh [1] and later applied to all accelerated fluids by Taylor [2]. Since then, this instability problem has been studied by several researchers under varying assumptions. The detailed description of other parameters of these investigations has been given by Chandrasekhar [3]. A theoretical model, for a class of viscoelastic fluids have been proposed by Oldroyd [4-6]. The Rayleigh-Taylor instability of a plane interfaces between viscous and viscoelastic fluid through a porous medium has been investigated by Sharma and Kumar [7]. Agrawal and Goel [8] have studied the instability of viscoelastic fluid in a porous medium. Bhatia and Mathur [9] have studied the instability of viscoelastic fluids in a vertical magnetic field through porous medium. Kumar et al. [10] have considered the instability of the plane interface between two viscoelastic superposed fluids in the presence of uniform rotation and variable magnetic field in porous medium.

In plasmas, RTI can occur when dense plasma is sup- ported against gravity by the pressure of the magnetic field. The investigation of Rayleigh-Taylor instabilities in magnetized plasma is a problem of considerable interest in space (ionospheric spread-F), fusion (curvature induced instabilities like interchange, ballooning, etc.) and the astrophysical plasmas. For a system of two incompressible plasma superimposed one over the other have been studied by Kalra and Talwar [11] and by Singh and Tandon [12]. Bhatia [13] studied the problem for a system of superimposed viscous plasmas in the presence of a horizontal magnetic field. The same previous system in the presence of uniform rotation was considered by Bhatia and Chhonkar [14] and by Sharma and Chhajlani [15]. The effects of Hall currents and viscosity on the Rayleigh-Taylor instability of incompressible infinitely conducting stratified plasma in the presence of horizontal magnetic field were studied by Ahsan and Bhatia [16]. The effects of Hall currents on the RTI of finitely conducting stratified partially ionized plasma in the presence of horizontal magnetic field is considered by Aiyub and Bhatia [17].

The linear growth rate of a finite layer plasma in which the density is continuously stratified exponentially along the vertical is studied by Goldston and Rutherford [18], in the presence of horizontal magnetic and rotation was studied by Bhatia and Sharma [19] and in the presence of horizontal magnetic field with the effect of a 
transverse velocity shear was studied by Wu et al. [20]. The effects of external magnetic field on RTI problem in inhomogeneous plasma rotating uniformly is studied by Al-Khateeb and Laham [21,22]. The effect of the velocity shear-induced viscosity gradient of low-frequency shear waves in viscoelastic dusty plasma was studied by Banerjee et al. [23]. The gravitational instability of rotating Walters $B^{\prime}$ viscoelastic partially ionized plasma permeated by an oblique magnetic field has been investigated in the presence of the effects of Hall currents by El-Sayed and Mohamed [24].

The hydrodynamic instabilities in quantum plasmas have been an important subject of research in the last a few years, where the quantum effects plays an important role in the behavior of the charged plasma particles when the de Broglie wavelength of the charge carriers becomes equal to or greater than the dimension of the quantum plasma system [25]. The quantum hydro-dynamic (QHD) model has been introduced by Gardner [26] for semiconductor physics to describe the transport of charge, momentum and energy in plasmas. The quantum magneto-hydrodynamic (QMHD) model was obtained by Haas [27] with the help of QHD model with magnetic field based on the Wigner-Maxwell equations. The Influence of quantum mechanism on the internal waves and the RTI in plasma is considered by Bychkov et al [28]. The effects of the quantum mechanism and magnetic field on RTI in ideal incompressible plasma are studied by Jintao et al. [29], where it plays a stability role on this problem. Quantum effects on RTI in inhomogeneous plasma rotating uniformly in an external magnetic field are investigated by Hoshoudy [30,31]. The effects of magnetic field on the RTI in quantum plasmas with para- and ferromagnetic properties have been considered by Mikhail et al. [32].The effect of quantum term on RTI of stratified plasma layer through porous medium is studied by Hoshoudy [33]. The RTI is investigated in a non-uniform dense quantum magneto-plasma by Ali et al. [34].

The purpose of this work is to examine theoretically the effect of the quantum mechanism on RTI for a finite thickness layer of incompressible viscoelastic plasma through porous media. This layer is confined between two rigid boundaries $(z=0, z=h)$. Using the normal mode approach, the dispersion relation is obtained analytically, and numerically analyzed.

\section{Fundamental Equations}

Our starting point is the set of equations of the quantum hydrodynamic (QHD) for an plasma model through a porous media (4-10 and 27-34)

$$
\begin{gathered}
\left\{1+\lambda \frac{\partial}{\partial t}\right\}\left\{\frac{\partial}{\partial t}+\frac{\boldsymbol{U}}{\varepsilon} \cdot \nabla\right\} \rho \frac{\boldsymbol{U}}{\varepsilon} \\
=\left\{1+\lambda \frac{\partial}{\partial t}\right\}\{-\nabla P+\rho \boldsymbol{g}+\boldsymbol{Q}\} \\
-\left\{1+\lambda_{0} \frac{\partial}{\partial t}\right\}\left\{\frac{\mu}{k_{1}} \boldsymbol{U}\right\} \\
\frac{\partial \rho}{\partial t}+\nabla \cdot(\rho \boldsymbol{U})=0
\end{gathered}
$$

Here $\boldsymbol{U}$ is the velocity of the fluid, $\rho$ is the density, $p$ thermal pressure, $\boldsymbol{g}$ is the gravitational acceleration, $1 / k_{1}$ is permeability of the medium and $\varepsilon$ is the porosity $(0<\varepsilon<1)$. Note that when $\varepsilon \rightarrow 1$ and $k_{1} \rightarrow \infty$ the system corresponds to a nonporous medium. $\mu$ is the viscosity of plasma. Here $\lambda$ is the stress relaxation time, $\lambda_{0}\left(\lambda_{0}<\lambda\right)$ is the strain retardation time.

$\boldsymbol{Q}=\frac{\hbar^{2}}{2 m_{e} m_{i}} \rho \nabla\left(\frac{\nabla^{2} \sqrt{\rho}}{\sqrt{\rho}}\right)$ is represented by Bohm Potential term (also, is called a quantum pressure and for more details on the derivation, see refs. [27,35]), where $\hbar$ is the Plank constant, $m_{e}$ is the electron mass and $m_{i}$ the ion mass.

To investigate the stability of hydrodynamic motion, we ask how the motion responds to a small fluctuation in the value of any of the flow variables appearing in the Euler equations. If the fluctuation grows in amplitude so that the flow never returns to its initial state, we say that the flow is unstable with respect to fluctuations of that type. Accordingly, we replace the variables in Equations (1) and (2) as in the form $\boldsymbol{U}=\boldsymbol{U}_{0}+\boldsymbol{U}_{1}, \rho=\rho_{0}+\rho_{1}$, $p=p_{0}+p_{1}$ and $\boldsymbol{Q}=\boldsymbol{Q}_{0}+\boldsymbol{Q}_{1}$. The quantities with subscripts " 0 " represent the unperturbed, or "zeroth-order" motion of the fluid, while the quantities with subscripts “ 1 ” represent a small perturbation about the zeroth-order quantities (first-order or linearized quantities); that is, $\left|U_{1}\right| \ll\left|U_{0}\right|,\left|\rho_{1}\right| \ll\left|\rho_{0}\right|,\left|p_{1}\right| \ll\left|p_{0}\right|$ and $\left|Q_{1}\right| \ll\left|Q_{0}\right|$. Substituting these expressions into Equations (1)-(4). In particular example of RTI we consider the fluid initially at rest. This means that $\boldsymbol{U}_{0}=0$, so the linearized equations can be easily derived from Equations (1) and (2) as

$$
\begin{aligned}
& \frac{\rho_{0}}{\varepsilon}\left\{1+\lambda \frac{\partial}{\partial t}\right\}\left\{\frac{\partial \boldsymbol{U}_{1}}{\partial t}\right\} \\
& =\left\{1+\lambda \frac{\partial}{\partial t}\right\}\left\{-\nabla p_{1}+\rho_{1} \boldsymbol{g}+\boldsymbol{Q}_{1}\right\} \\
& \quad-\left\{1+\lambda_{0} \frac{\partial}{\partial t}\right\}\left\{\frac{\mu}{k_{1}} \boldsymbol{U}_{1}\right\}
\end{aligned}
$$




$$
\begin{gathered}
\frac{\partial \rho_{1}}{\partial t}+\nabla \cdot\left(\rho_{0} \boldsymbol{U}_{1}\right)=0 \\
\boldsymbol{Q}_{1}=\frac{\hbar^{2}}{2 m_{e} m_{i}}\left\{\frac{1}{2} \nabla\left(\nabla^{2} \rho_{1}\right)-\frac{1}{2 \rho_{0}} \nabla \rho_{1} \nabla^{2} \rho_{0}-\frac{1}{2 \rho_{0}} \nabla \rho_{0} \nabla^{2} \rho_{1}\right. \\
+\frac{\rho_{1}}{2 \rho_{0}^{2}} \nabla \rho_{0} \nabla^{2} \rho_{0}-\frac{1}{2 \rho_{0}} \nabla\left(\nabla \rho_{0} . \nabla \rho_{1}\right) \\
+\frac{\rho_{1}}{4 \rho_{0}^{2}} \nabla\left(\nabla \rho_{0}\right)^{2}+\frac{1}{2 \rho_{0}^{2}}\left(\nabla \rho_{0}\right)^{2} \nabla \rho_{1} \\
\left.+\frac{1}{\rho_{0}^{2}}\left(\nabla \rho_{0} . \nabla \rho_{1}\right) \nabla \rho_{0}-\frac{\rho_{1}}{\rho_{0}^{3}}\left(\nabla \rho_{0}\right)^{3}\right\}
\end{gathered}
$$

We now appeal to the fact that, for many situations of interest in ICF (inertial-confinement fusion), unstable flow occurs at velocities much smaller than the local sound speed. This has the effect that accelerations in the flow are not strong enough to change the density of a fluid element significantly, so the fluid moves without compressing or expanding. In such a situation we call the flow incompressible. Provided that we are well away from shock waves or centers of convergence, the assumption of incompressible flow is often valid. To say that fluid elements move without changing density is to say that the Lagrangian total derivative of density is zero, that

$$
\frac{\mathrm{d} \rho}{\mathrm{d} t}=\left(\frac{\partial}{\partial t}+\boldsymbol{U} \cdot \nabla\right) \rho=0
$$

We also linearize this equation, where the first-order quantities, as before become

$$
\frac{\partial \rho_{1}}{\partial t}+\left(\boldsymbol{U}_{1} \cdot \nabla\right) \rho_{0}=0
$$

Comparing this equation to Equation(4), which can be rewritten in expanded form as

$$
\frac{\partial \rho_{1}}{\partial t}+\left(\boldsymbol{U}_{1} \cdot \nabla\right) \rho_{0}+\rho_{0} \nabla \cdot \boldsymbol{U}_{1}=0
$$

we see that subtracting Equation (7) from Equation (8) yields

$$
\nabla \cdot \boldsymbol{U}_{1}=0
$$

This is a consequence of the assumption of incompressible flow. So, we can use either Equation (7) or Equation (9) to replace the linearized continuity Equation (4) under this assumption. One can seen that the set of Equations (3), (7) and (9) is complete for describing the quantum effects on the Rayleigh-Taylor instability of incompressible plasma. Now, where

$$
\boldsymbol{U}_{1}=\left(u_{x 1}, u_{y 1}, u_{z 1}\right), \quad \boldsymbol{g}=(0,0,-g), \quad \boldsymbol{Q}_{1}=\left(Q_{x 1}, Q_{y 1}, Q_{z 1}\right)
$$

The fluid is arranged in horizontal strata, and then $\rho_{0}$ is a function of the vertical coordinate $\left(\rho_{0}=\rho_{0}(z)\right)$ and $p_{0}=p_{0}(z)$.Then, the system Equations (3), (7) and (9) become as in the appendix (Equations (30)-(37)).

Considering that the perturbation in any physical quantity takes the form

$$
\psi_{1}(x, y, z, t)=\psi_{1}(z) \exp \left(i\left(k_{x} x+k_{y} y-\omega t\right)\right)
$$

where $k_{x}$ and $k_{y}$ are horizontal components of the wave-number vector $\boldsymbol{k}$ such that $k^{2}=k_{x}^{2}+k_{y}^{2}$ and $\omega$ (may be complex $\left(\omega=\omega_{r}+i \gamma\right)$ ) is the frequency of perturbations or the rate at which the system departs from equilibrium thee initial state. Using the expression (10) in the system of equations (30)-(37) in the appendix, they become as in the appendix (see Equations (38)-(45))

Now, eliminating some of the variables from the system of Equations (38)-(42) in the appendix, we have

$$
\begin{aligned}
& {\left[\rho_{0} k^{2}\left\{(-i \omega)+\frac{\varepsilon v_{0}}{k_{1}} \frac{1-\lambda_{0}(i \omega)}{1-\lambda(i \omega)}\right\} u_{z 1}\right]} \\
& -\left\{(-i \omega)+\frac{\varepsilon v_{0}}{k_{1}} \frac{1-\lambda_{0}(i \omega)}{1-\lambda(i \omega)}\right\} \frac{\mathrm{d}}{\mathrm{d} z}\left(\rho_{0} \frac{\mathrm{d} u_{z 1}}{\mathrm{~d} z}\right) \\
& +\frac{g k^{2}}{i \omega}\left(\frac{\mathrm{d} \rho_{0}}{\mathrm{~d} z}\right) u_{z 1}+\frac{k^{2}}{i \omega}\left\{\frac{\hbar^{2}}{4 m_{e} m_{i}}\right\} \\
& \cdot\left\{\frac{1}{\rho_{0}}\left(\frac{\mathrm{d} \rho_{0}}{\mathrm{~d} z}\right)^{2} \frac{\mathrm{d}^{2} u_{z 1}}{\mathrm{~d} z^{2}}-\frac{1}{\rho_{0}^{2}}\left(\frac{\mathrm{d} \rho_{0}}{\mathrm{~d} z}\right)\left(\begin{array}{l}
\left(\frac{\mathrm{d} \rho_{0}}{\mathrm{~d} z}\right)^{2} \\
-2 \rho_{0} \frac{\mathrm{d}^{2} \rho_{0}}{\mathrm{~d} z^{2}}
\end{array}\right) \frac{\mathrm{d} u_{z 1}}{\mathrm{~d} z}\right. \\
& \left.-\frac{k^{2}}{\rho_{0}}\left(\frac{\mathrm{d} \rho_{0}}{d z}\right)^{2} u_{z 1}\right\}=0
\end{aligned}
$$

and if we consider,

$$
\omega_{*}=(i \omega)\left\{(-i \omega)+\frac{\varepsilon v_{0}}{k_{1}} \frac{1-\lambda_{0}(i \omega)}{1-\lambda(i \omega)}\right\}
$$

Then Equation (11) becomes

$$
\begin{aligned}
& {\left[\rho_{0} \omega_{*}+k^{2} A\right] \frac{\mathrm{d}^{2} u_{z 1}}{\mathrm{~d} z^{2}}+\left[\omega_{*}\left(\frac{\mathrm{d} \rho_{0}}{\mathrm{~d} z}\right)+k^{2} B\right] \frac{\mathrm{d} u_{z 1}}{\mathrm{~d} z}} \\
& -k^{2}\left[\rho_{0} \omega_{*}-(C-g) \frac{\mathrm{d} \rho_{0}}{\mathrm{~d} z}\right] u_{z 1}=0
\end{aligned}
$$

where 


$$
\begin{aligned}
& A=-\frac{\hbar^{2}}{4 m_{e} m_{i}} \frac{1}{\rho_{0}}\left(\frac{\mathrm{d} \rho_{0}}{\mathrm{~d} z}\right)^{2}, \\
& B=\frac{\hbar^{2}}{4 m_{e} m_{i}} \frac{1}{\rho_{0}^{2}}\left(\frac{\mathrm{d} \rho_{0}}{\mathrm{~d} z}\right)^{2}\left\{\left(\frac{\mathrm{d} \rho_{0}}{\mathrm{~d} z}\right)^{2}-2 \rho_{0} \frac{\mathrm{d}^{2} \rho_{0}}{\mathrm{dz}^{2}}\right\}, \\
& C=\frac{\hbar^{2}}{4 m_{e} m_{i}} \frac{k^{2}}{\rho_{0}}\left(\frac{\mathrm{d} \rho_{0}}{\mathrm{~d} z}\right) .
\end{aligned}
$$

\section{A Continuously Stratified Plasma Layer}

For a finite thickness layer of incompressible plasma through porous media bounded on the other interface by a rigid boundary at $z=0, \quad z=h$ and

$\rho_{0}(z)=\rho_{0}(0) \exp \left(z / L_{D}\right)$, Equation (13) takes the form

$$
\begin{aligned}
& {\left[\omega_{*}-\omega_{q}^{2}\right] \frac{\mathrm{d}^{2} u_{z 1}}{\mathrm{~d} z^{2}}+\frac{1}{L_{D}}\left[\omega_{*}-\omega_{q}^{2}\right] \frac{\mathrm{d} u_{z 1}}{\mathrm{~d} z}} \\
& -k^{2}\left\{\left[\omega_{*}-\omega_{q}^{2}\right]+\frac{g}{L_{D}}\right\} u_{z 1}=0
\end{aligned}
$$

where $\omega_{q}^{2}=\frac{\hbar^{2} k^{2}}{4 L_{D}^{2} m_{e} m_{i}}$.

Now, we put the solution of Equation (15) in the form $u_{z 1}=\sin \left(\frac{n \pi}{h} z\right) \exp (\lambda z)$ and substituting into Equation (15), the dispersion relation may be written as

$$
\begin{gathered}
\omega_{*}=\omega_{q}^{2}-\frac{4 g k^{2} h^{2} L_{D}}{h^{2}+4 k^{2} h^{2} L_{D}^{2}+4 n^{2} \pi^{2} L_{D}^{2}} \\
\lambda=-\frac{1}{2 L_{D}}
\end{gathered}
$$

Now, we insert the following dimensionless quantities in Equation (16)

$$
\begin{gathered}
\omega^{*^{2}}=\frac{\omega^{2}}{\omega_{p e}^{2}}, \omega_{q}^{*^{2}}=\frac{\hbar^{2}}{4 L_{D}^{4} m_{e} m_{i} \omega_{p e}^{2}}, \omega_{\varepsilon}^{*}=\frac{\varepsilon}{\omega_{p e}}, \\
\omega_{v_{0}}^{*}=\frac{v_{0}}{\omega_{p e}}, \omega_{k_{1}}^{*}=\frac{k_{1}}{\omega_{p e}}, \omega_{\lambda_{0}}^{*}=\lambda_{0} \omega_{p e}, \omega_{\lambda}^{*}=\lambda \omega_{p e} \\
h^{* 2}=\frac{h^{2}}{L_{D}^{2}}, \quad k^{*^{2}}=k^{2} L_{D}^{2}, g^{*}=\frac{g}{\omega_{p e}^{2} L_{D}}
\end{gathered}
$$

where $\omega_{p e}=\left(\frac{\rho_{0} e^{2}}{m_{e}^{2} \varepsilon_{0}}\right)^{1 / 2}$ is the plasma frequency, and use Equation (12), then the dispersion relation (16) takes the form

$$
a_{1}\left(i \omega^{*}\right)^{3}+a_{2}\left(i \omega^{*}\right)^{2}+a_{3}\left(i \omega^{*}\right)^{2}+a_{4}=0
$$

$$
\begin{gathered}
a_{1}=\frac{\omega_{\varepsilon}^{*} \omega_{v_{0}}^{*} \omega_{\lambda_{0}}^{*} \omega_{\lambda}^{*}}{\omega_{k_{1}}^{*}} \\
a_{2}=1+\frac{\omega_{\varepsilon}^{*} \omega_{v_{0}}^{*}}{\omega_{k_{1}}^{*}}\left(\omega_{\lambda_{0}}^{*}-\omega_{\lambda}^{*}\right) \\
-\omega_{\lambda}^{* 2}\left(k^{* 2} \omega_{q}^{* 2}-\frac{4 g^{*} k^{* 2} h^{* 2}}{h^{* 2}+4 k^{* 2} h^{* 2}+4 n^{2} \pi^{2}}\right) \\
a_{3}=-\frac{\omega_{\varepsilon}^{*} \omega_{v_{0}}^{*}}{\omega_{k_{1}}^{*}} \\
a_{4}=\left(k^{* 2} \omega_{q}^{* 2}-\frac{4 g^{*} k^{* 2} h^{* 2}}{h^{* 2}+4 k^{* 2} h^{* 2}+4 n^{2} \pi^{2}}\right)
\end{gathered}
$$

From the system of Equations (17)-(21), it is clear that, the effect of strain retardation time $\lambda_{0}^{*}$ is connected by the presence of the resistance term (i.e., the effect of $\lambda_{0}^{*}$ will occur in the presence of $\left.\omega_{\varepsilon}^{*}, \omega_{v_{0}}^{*}, \omega_{k_{1}}^{*}\right)$, while the effect of stress relaxation time $\lambda^{*}$ may be occur alone or it is connected by the presence of $\omega_{\varepsilon}^{*}, \omega_{v_{0}}^{*}, \omega_{k_{1}}^{*}$ and $\omega_{q}^{*}$.

Now, where $\omega^{*}=\omega_{r}^{*}+i \gamma$ and in the case of $\omega_{r}^{*}=0$ and $\gamma \neq 0$ (stable oscillations), then Equation (17) becomes

$$
a_{1} \gamma^{3}-a_{2} \gamma^{2}+a_{3} \gamma-a_{4}=0
$$

\section{Special Cases}

Here, some special cases are considered from Equation (22) to clarify the different roles of the parameter's problem.

1) At $\omega_{\varepsilon}^{*}=0, \omega_{v_{0}}^{*}=0, \omega_{\lambda_{0}}^{*}=0, \omega_{\lambda}^{*}=0, \omega_{q}^{*}=0$, we find that $a_{1}=a_{3}=0, a_{2}=1$ and

$$
a_{4}=-\frac{4 g^{*} k^{* 2} h^{* 2}}{h^{* 2}+4 k^{* 2} h^{* 2}+4 n^{2} \pi^{2}}
$$

So the normalized growth rate from Equation (22) is

$$
\gamma_{\text {classical }}=\sqrt{\frac{4 g^{*} k^{* 2} h^{* 2}}{h^{* 2}+4 k^{* 2} h^{* 2}+4 n^{2} \pi^{2}}}
$$

This case is considered by Goldston and Rutherford (see ref. [18]), where the system represents an exponentially growing perturbation.

2) At $\omega_{\varepsilon}^{*}=0, \omega_{v_{0}}^{*}=0, \omega_{\lambda_{0}}^{*}=0, \omega_{\lambda}^{*}=0, \omega_{q}^{*} \neq 0$, we find that $a_{1}=a_{3}=0, a_{2}=1$, while $a_{4}$ is as in Equation (21). So the normalized growth rate from Equation (22) is

$$
\gamma_{\text {quantum }}=\sqrt{\frac{4 g^{*} k^{* 2} h^{* 2}}{h^{* 2}+4 k^{* 2} h^{* 2}+4 n^{2} \pi^{2}}-k^{* 2} \omega_{q}^{* 2}}
$$


This case is studied in Refs. [29-33]. It is clarified that, the quantum term has stabilizing effect on RTI problem. This influence is obvious from Equations (23) and (24), where $\gamma_{\text {quantum }}<\gamma_{\text {classical }}$ for all the values of wave number. Also, the system increases as $k^{*}$ increases through the range $0<k^{*}<k_{\max }^{*}$, when $k^{*}>k_{\max }^{*}$ it starts to decreases as $k^{*}$ increases, where

$$
k_{\max }^{*}=\sqrt{\frac{1}{\omega_{q}^{*}}\left(\sqrt{g^{*}}-1\right)\left(\frac{1}{4}+\left(\frac{n \pi}{h^{*}}\right)^{2}\right)}
$$

and at $k^{*}=k_{c}^{*}$ the system arrives to complete stability with $k_{c}^{*}=\sqrt{\frac{g^{*}}{\omega_{q}^{*^{2}}}-\left(\frac{1}{4}+\left(\frac{n \pi}{h^{*}}\right)^{2}\right)}$.

3) At $\omega_{\varepsilon}^{*}=0, \omega_{v_{0}}^{*}=0, \omega_{\lambda_{0}}^{*}=0, \omega_{\lambda}^{*} \neq 0, \omega_{q}^{*}=0$, we find that $a_{1}=a_{3}=0$, while

$$
a_{2}=1+\frac{4 g^{*} k^{* 2} h^{* 2}}{h^{* 2}+4 k^{* 2} h^{* 2}+4 n^{2} \pi^{2}} \omega_{\lambda}^{* 2}
$$

and

$$
a_{4}=-\frac{4 g^{*} k^{* 2} h^{* 2}}{h^{* 2}+4 k^{* 2} h^{* 2}+4 n^{2} \pi^{2}}
$$

Then the normalized growth rate from Equation (22) is given by

$$
\gamma_{\text {stress relaxation }}=\sqrt{\frac{\frac{4 g^{*} k^{* 2} h^{* 2}}{h^{* 2}+4 k^{* 2} h^{* 2}+4 n^{2} \pi^{2}}}{\frac{4 g^{*} k^{* 2} h^{* 2}}{h^{* 2}+4 k^{* 2} h^{* 2}+4 n^{2} \pi^{2}} \omega_{\lambda}^{* 2}}}
$$

Also, from Equations (23) and (25) one can see that, the stress relaxation time has a stabilizing role on the given system. This role increases with increasing of the magnitudes of stress relaxation time. While in the presence of quantum term the normalized growth rate in Equation (25) takes the form

$$
\begin{aligned}
& \gamma_{\text {quantum, stress relaxation }} \\
& =\sqrt{\frac{4 g^{*} k^{* 2} h^{* 2}}{h^{* 2}+4 k^{* 2} h^{* 2}+4 n^{2} \pi^{2}}-k^{* 2} \omega_{q}^{* 2}} \\
& \left.\frac{4 g^{*} k^{* 2} h^{* 2}}{h^{* 2}+4 k^{* 2} h^{* 2}+4 n^{2} \pi^{2}}-k^{* 2} \omega_{q}^{* 2}\right] \omega_{\lambda}^{* 2}
\end{aligned}
$$

from above results it very clear that

$$
\gamma_{\text {quantum, stress relaxation }}<\gamma_{\text {stress relaxation }}<\gamma_{\text {classical }}
$$

4) At $\omega_{\varepsilon}^{*} \neq 0, \omega_{v_{0}}^{*} \neq 0, \omega_{\lambda_{0}}^{*}=0, \omega_{\lambda}^{*}=0, \omega_{q}^{*} \neq 0$, we find that $a_{1}=0, a_{2}=1$, while $a_{3}, a_{4}$ are as in Equations (20) and (21). So the normalized growth rate given by

$$
\gamma^{2}+\frac{\omega_{\varepsilon}^{*} \omega_{v_{0}}^{*}}{\omega_{k_{1}}^{*}} \gamma+\left(k^{* 2} \omega_{q}^{* 2}-\frac{4 g^{*} k^{* 2} h^{* 2}}{h^{* 2}+4 k^{* 2} h^{* 2}+4 n^{2} \pi^{2}}\right)=0
$$

corresponds Equation (33) ref. [33], where the normalized growth rate given by

$$
\begin{aligned}
& \gamma_{\text {porous medium }} \\
& =-\frac{\omega_{\varepsilon}^{*} \omega_{v_{0}}^{*}}{\omega_{k_{1}}^{*}} \\
& \pm \sqrt{\left(\frac{\omega_{\varepsilon}^{*} \omega_{v_{0}}^{*}}{\omega_{k_{1}}^{*}}\right)^{2}+4\left(\frac{4 g^{*} k^{* 2} h^{* 2}}{h^{* 2}+4 k^{* 2} h^{* 2}+4 n^{2} \pi^{2}}-k^{* 2} \omega_{q}^{* 2}\right)}
\end{aligned}
$$

and in the presence of the strain retardation, Equation(28) becomes

$$
\begin{aligned}
& \gamma_{\text {porous medium, strain retardation }} \\
& =\frac{-\omega_{\varepsilon}^{*} \omega_{v_{0}}^{*}}{\omega_{k_{1}}^{*}+\omega_{\varepsilon}^{*} \omega_{v_{0}}^{*} \omega_{\lambda_{0}}^{*}} \pm \sqrt{\left(\frac{\omega_{\varepsilon}^{*} \omega_{v_{0}}^{*}}{\omega_{k_{1}}^{*}+\omega_{\varepsilon}^{*} \omega_{v_{0}}^{*} \omega_{\lambda_{0}}^{*}}\right)^{2}+\frac{4 \omega_{\varepsilon}^{*} \omega_{v_{0}}^{*}}{\omega_{k_{1}}^{*}+\omega_{\varepsilon}^{*} \omega_{v_{0}}^{*} \omega_{\lambda_{0}}^{*}}} \\
& \times\left(\frac{4 g^{*} k^{* 2} h^{* 2}}{h^{* 2}+4 k^{* 2} h^{* 2}+4 n^{2} \pi^{2}}-k^{* 2} \omega_{q}^{* 2}\right)
\end{aligned}
$$

From Equations (28) and (29) one can see that, the stabilizing role that plays the stress strain retardation time on the given system.

In the general case, if we wish to look into the effect of various factors, on the instability of the considered system, Equation (22) is to be numerically solved. Pertaining results are presented in Figures 1-3.

Figure 1 shows the effect of the factors $\omega_{q}^{*}=0.8$, $\omega_{\varepsilon}^{*}=0.1, \omega_{k_{1}}^{*}=0.1, \omega_{\lambda_{0}}^{*}=0.3$ and $\omega_{\lambda}^{*}=0.5$ every one is alone, where the square normalized growth rate $\gamma^{2}$ is plotted against the square normalized wave number $k^{* 2}$. One can see that, in the absence of $\omega_{q}^{*}=0$ the magnitudes of $\gamma^{2}$ in the presence of the factors $\omega_{\varepsilon}^{*}, \omega_{k_{1}}^{*}, \omega_{\lambda_{0}}^{*}$ and $\omega_{\lambda}^{*}$ are less than their magnitudes in the classical case, which means that these factors have a stabilizing effect on the considered system. Also, it can be seen that, no mode of maximum instability exists when $\omega_{q}^{*}=0$ as the square normalized growth rate $\gamma^{2}$ usually increases by increase with the square normalized wave number values. While, in the presence of quantum term $\omega_{q}^{*}=0.8$ (the detailed description of this case is given in the next part), there is a mode of maximum instability, where the square normalized growth rate $\gamma^{2}$ increases with $k^{*^{2}}$ increases through the range $0<k^{*^{2}}<k_{\max }^{*^{2}}$ (at $k_{\max }^{*^{2}}$ the square normalized growth rate arrives to the maximum 


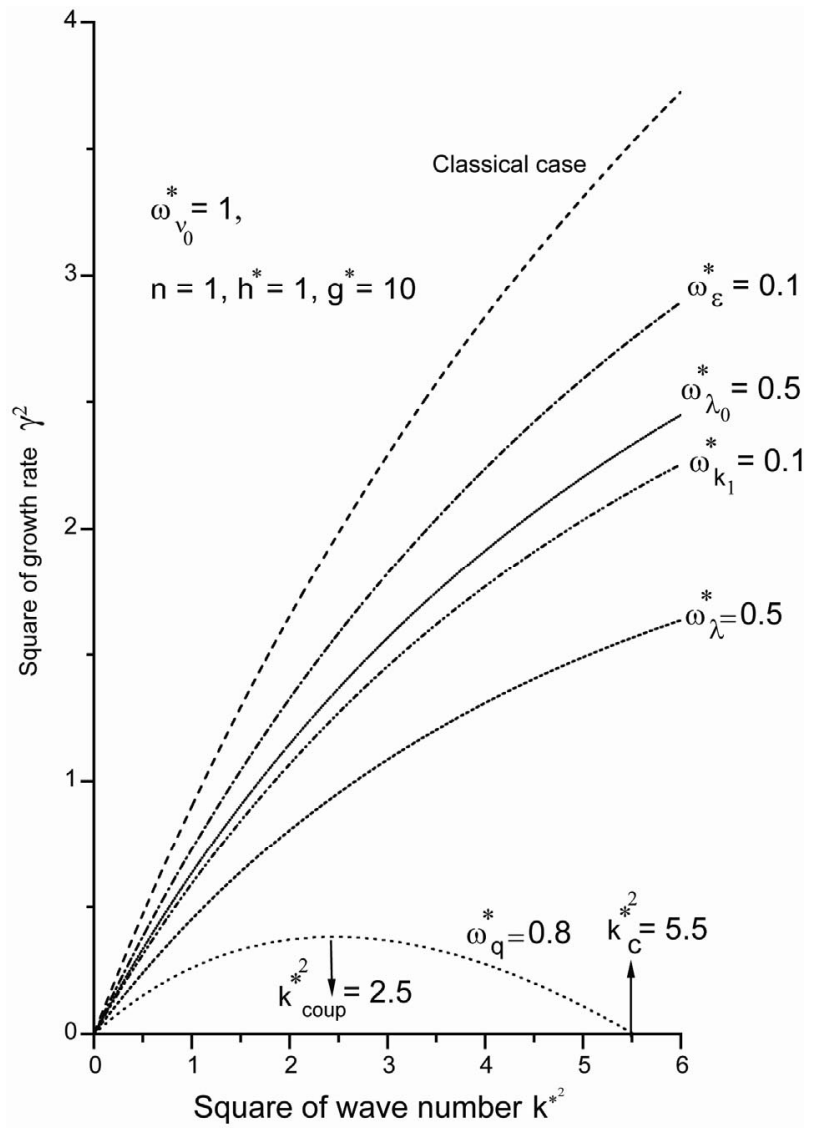

Figure 1. The square normalized growth rate $\gamma^{2}$ against the square normalized wave number $\boldsymbol{k}^{* 2}$, where the role of the different parameters are separated occurred at $n=1, h^{*}=1$ and $g^{*}=10$.

instability, for our example in Figure 1 and at $\omega_{q}^{*}=0.8$ we note $k_{\max }^{*^{2}}=2.5$ ), and when $k^{*^{2}}>k_{\max }^{*^{2}}$ the square normalized growth rate $\gamma^{2}$ starts to decreases as $k^{*^{2}}$ increases and then goes to the complete stable at $k_{c}^{*^{2}}=5.5\left(k_{c}^{*^{2}}\right.$ is the critical value for stability, at this point the square normalized growth rate goes to zero). This means that the quantum term has a crucial capability to suppress the instability, while the other factors have not liked this strength.

The role of porosity of the porous medium, the medium permeability, kinematic viscosity with quantum term is explained in ref. [33]. So, our next numerical results concentrate on the role of both strain retardation and stress relaxation.

Figures $\mathbf{2}$ and $\mathbf{3}$ are plotted to indicate the influence of the strain retardation time $\lambda_{0}^{*}$ and the stress relaxation time $\lambda^{*}$ on the problem, respectively, in the presence of quantum effect $\omega_{q}^{*}=0.8$. Figure 2 shows that the presence of $\lambda_{0}^{*}$ does not effect on both the maximum point $k_{\max }^{*^{2}}$ for the instability and the corresponding critical point $k_{c}^{*^{2}}$ for the stability, where $k_{\max }^{*^{2}}=2.5$ and $k_{c}^{*^{2}}=5.5$

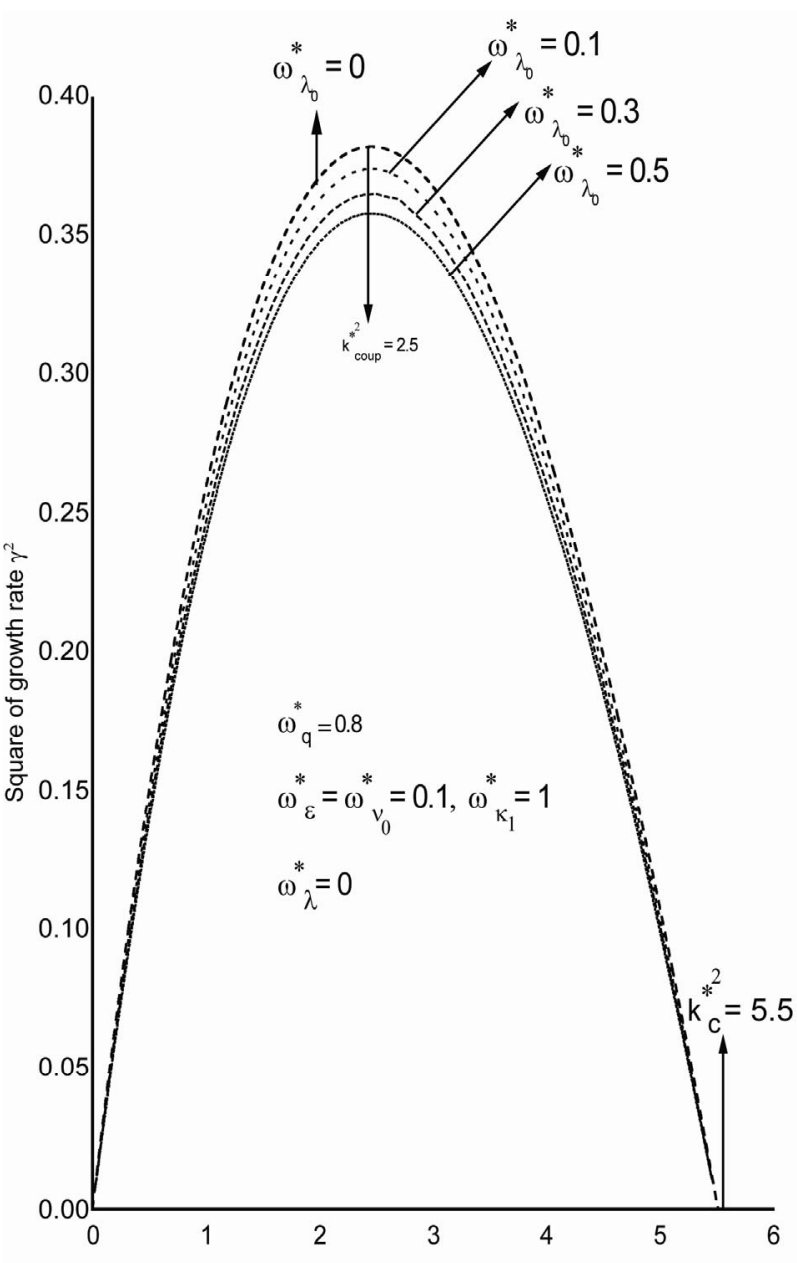

Square of wave number $k^{*^{2}}$

Figure 2. The role of the strain retardation time $\lambda_{0}^{*}(0.1,0.3,0.5)$ with the parameters $\omega_{q}^{*}=0.8, \omega_{\varepsilon}^{*}=\omega_{v_{0}}^{*}=0.1, \omega_{k_{1}}^{*}=1$, $\lambda^{*}=0, n=1, h^{*}=1$ and $g^{*}=10$.

at $\lambda_{0}^{*}(0.1,0.3,0.5)$. The same note for the stress relaxation time $\lambda^{*}(0.1,0.3,0.5)$ is shown in Figure 3. However, the magnitudes of $\gamma^{2}$ in the presence of both $\omega_{\lambda_{0}}^{*}$ and $\omega_{\lambda}^{*}$ are less than their magnitudes in the presence of the quantum term alone, which implies that both $\omega_{\lambda_{0}}^{*}$ and $\omega_{\lambda}^{*}$ have a stabilizing role on the considered system. For the same values, the effect of the stress relaxation time $\omega_{\lambda}^{*}$ is greater than the effect of the strain retardation time $\omega_{\lambda_{0}}^{*}$ on the considered system.

\section{Conclusions}

The effect of quantum term on the Rayleigh-Taylor instability of stratified fluid/plasma through porous media has been studied. The effect of elasticity is revealed 


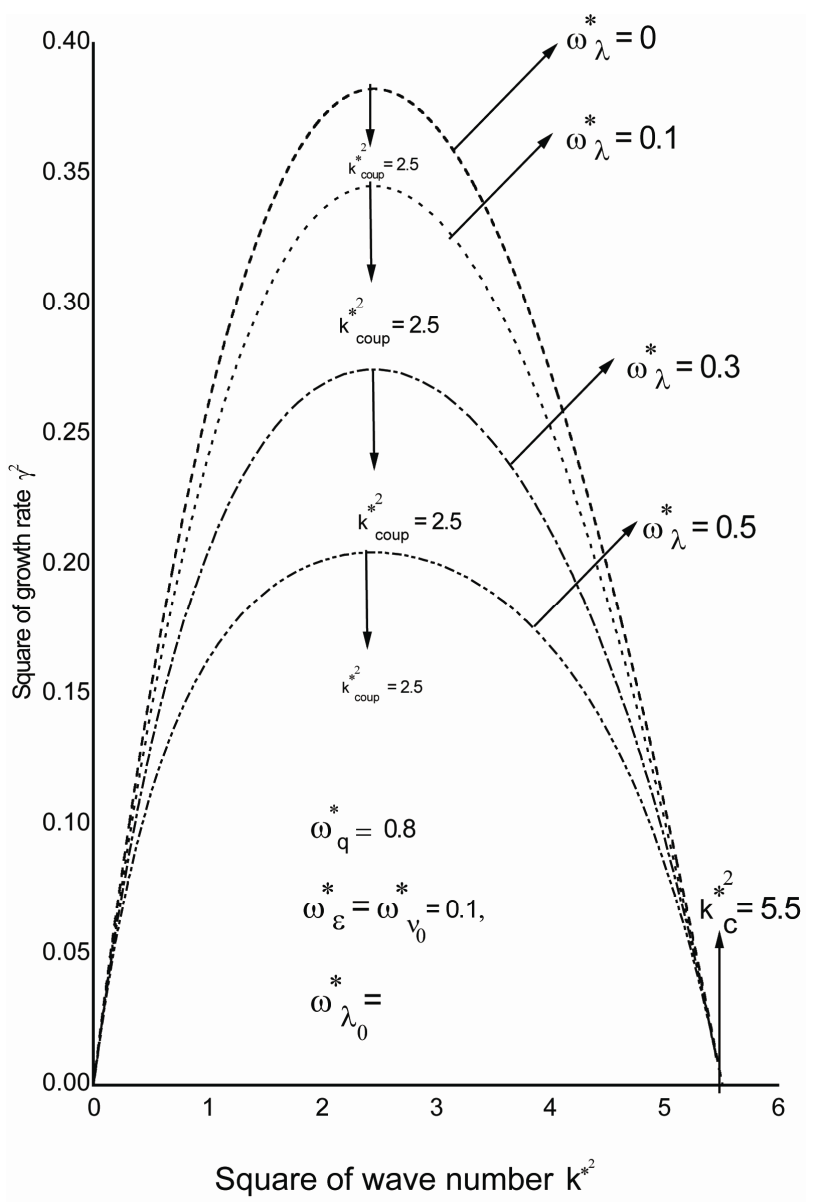

Figure 3. The role of the stress relaxation time $\lambda^{*}(0.1,0.3,0.5)$ with the parameters $\omega_{q}^{*}=0.8, \omega_{\varepsilon}^{*}=\omega_{v_{0}}^{*}=0.1, \omega_{k_{1}}^{*}=1, \lambda_{0}^{*}=0$, $n=1, h^{*}=1$ and $g^{*}=10$.

through the strain retardation time and the stress relaxation time. It is found that, the critical point for the stability $k_{c}^{*^{2}}$ that occurs in the presence of quantum term remains unchanged by the addition of the other parameters of the problem. Both maximum $k_{\max }^{*}$ and critical $k_{c}^{*}$ point for the instability are unchanged by the addition of the strain retardation and the stress relaxation. All growth rates are reduced in the presence of porosity of the medium, the medium permeability, the strain retardation time and the stress relaxation time. These results indicate that quantum effect plays a major role in securing a complete stability for the system at hand, while other parameters are only of secondary significance.

Finally, our select model is more stable than those considered in refs. [29-33]. This discrepancy highlights a stabilizing role due to the presence of both the strain retardation and the stress relaxation on Rayleigh-Taylor instability problem, increasing the dissipation of any disturbance, thus providing an increased stability.

\section{Acknowledgements}

The author would like to thank the referees for their useful suggestions and comments that improved the original manuscript.

\section{References}

[1] L. Rayleigh, "Investigation of the Character of the Equilibrium of an Incompressible Heavy Fluid of Variable Density," Proceedings of the London Mathematical Society, Vol. 14, 1882, pp. 170-177. doi:10.1112/plms/s1-14.1.170

[2] G. I. Taylor, "The Instability of Liquid Surfaces When Accelerated in a Direction Perpendicular to Their Planes," Proceedings of the Royal Society of London. Series A, Vol. 201, 1950, pp. 192-196. doi:10.1098/rspa.1950.0052

[3] S. Chandrasekhar, "Hydrodynamic and Hydromagnetic Stability,” Dover, New York, 1961.

[4] J. G. Oldroyd, "On the Formulation of Rheological Equations of State," Proceedings of the Royal Society of London. Series A, Vol. 200, 1950, pp. 523-541. doi:10.1098/rspa.1950.0035

[5] J. G. Oldroyd, "The Elastic and Viscous Properties of Emulsions and Suspensions," Proceedings of the Royal Society of London. Series A, Vol. 218, No. 1132, 1953, pp. 122-132. doi:10.1098/rspa.1953.0092

[6] J. G. Oldroyd, “Non-Newtonian Effects in Steady Motion of Some Idealized Elastico-Viscous Liquids," Proceedings of the Royal Society of London. Series A, Vol. 245, No. 1241, 1958, pp. 278-279. doi:10.1098/rspa.1958.0083

[7] R. C. Sharma and P. Kumar, "Rayleigh-Taylor Instability of Viscous-Viscoelastic Fluids through Porous Medium," Indian Journal of Pure and Applied Mathematics, Vol. 24, No. 9, 1993, pp. 563-569.

[8] S. C. Agrawal and A. K. Goel, "Shear Flow Instability of Viscoelastic Fluid in a Porous Medium,” Indian Journal of Pure and Applied Mathematics, Vol. 29, 1998, pp. 969-981.

[9] P. K. Bhatia and R. P Mathur, "Instability of Viscoelastic Superposed Fluids in a Vertical Magnetic Field through Porous Medium,” Ganita Sandesh (India), Vol. 17, 2003, pp. 21-32.

[10] P. Kumar, H. Mohan and G. J. Singh, "Rayleigh—-Taylor Instability of Rotating Oldroydian Viscoelastic Fluids in Porous Medium in Presence of a Variable Magnetic Field," Transport in Porous Media, Vol. 56, No. 2, 2004, pp. 19-208. doi:10.1023/B:TIPM.0000021828.01346.57

[11] G. L. Kalra and S. P. Talwar, "Slipping Stream Instability of a Self-Gravitating Hydromagnetic Gas Cloud," Monthly Notices of the Royal Astronomical Society, Vol. 135, 1967, pp. 391-398.

[12] S. Singh and J. N. Tnadon, "On the Rayleigh-Taylor Instability in Hydromagnetics with Finite Electrical Resistivity and Hall Current,” Journal of Plasma Physics, Vol. 
3, No. 4, 1969, pp. 633-642. doi:10.1017/S0022377800004670

[13] P. K. Bhatia and IL Nuovo Cimento, "Rayleigh-Taylor Instability of Viscous Superposed Conducting Fluids," Il Nuovo Cimento B (1971-1996), Vol. 19 B, No. 2, 1974, pp. 161-168.

[14] P. K. Bhatia and R. P. S. Chhonkar, "Rayleigh-Taylor Instability of Two Viscous Superposed Rotating and Conducting Fluids,” Astrophysics and Space Science, Vol. 114, No. 2, 1985, pp. 271-276. doi:10.1007/BF00653970

[15] P. K. Sharma and R. K. Chhajlani, "Effect of Rotation on the Rayleigh-Taylor Instability of Superposed Magnetic Conducting Plasma," Physics of plasmas, Vol. 5, No. 6, 1998, pp. 2203-2209. doi:10.1063/1.872893

[16] A. Ali and P. K. Bhatia, "Rayleigh-Taylor Instability of a Stratified Hall Plasma in Two-Dimensional Horizontal Magnetic Field,” Physica Scripta, Vol. 47, 1993, pp. 567 -570. doi:10.1088/0031-8949/47/4/016

[17] A. Khan and P. K. Bhatia, "Gravitational Instability of a Rotating Fluid in an Oblique Magnetic Field," Physica Scripta, Vol. 47, 1993, pp. 230-234. doi:10.1088/0031-8949/47/2/018

[18] R. J. Goldston and P. H. Rutherford, "Introduction to Plasma Physics,” Institute of Physics, London, 1997.

[19] P. K. Bhatia and A. Sharma, "Rayleigh-Taylor Instability of a Stratified Fluid/Plasma in an Inhomogeneous Magnetic Field," Proceedings of the National Academy of Sciences, India. Section A. Physical, Vol. 68 (A) III, 1998, pp. 239-247.

[20] Z. Wu, W. Zhang, D. Li and W. Yang, "Effect of Magnetic Field and Equilibrium Flow on Rayleigh-Taylor Instability," Chinese Physics Letters, Vol. 21, No. 10, 2004, pp. 2001-2004. doi:10.1088/0256-307X/21/10/038

[21] A. M. Al-Khateeb and N. M. Laham, "Magnetic Field Effects on the Rayleigh-Taylor Instability in Inhomogeneous Plasma,” The Arabian Journal for Science and Engineering, Vol. 27, No. 1A, 2002, pp. 75-83.

[22] A. M. Al-Khateeb and N. M. Laham, "Stability Criteria of Rayleigh-Taylor Modes in Magnetized Plasmas," Contributions to Plasma Physics, Vol. 43, No. 1, 2003, pp. 25-32. doi:10.1002/ctpp.200310003

[23] D. Banerjee M. S. Janaki, N. Chakrabarti and M. Chaudhuri, "Viscosity Gradient-Driven Instability of "Shear Mode' in a Strongly Coupled Plasma," New Journal of Physics, Vol. 12, 2010, p. 123031. doi:10.1088/1367-2630/12/12/123031

[24] M. F. El-Sayed and R. A. Mohamed, "Gravitational In- stability of Rotating Viscoelastic Partially Ionized Plasma in the Presence of an Oblique Magnetic Field and Hall Current," International Scholarly Research Network ISRN Mechanical Engineering, 2011, Article ID 597172.

[25] G. Manfredi, "How to Model Quantum Plasmas," Fields Institute Communications Series, Vol. 46, 2005, pp. 263287.

[26] C. Gardner, "The Quantum Hydrodynamic Model for Semiconductor Devices," SIAM Journal on Applied Mathematics, Vol. 54, 1994, pp. 409-427. doi:10.1137/S0036139992240425

[27] F. Haas, “Quantum Magnetohydrodynamics," Physics of Plasmas, Vol. 12, 2005, p. 062117. doi:10.1063/1.1939947

[28] B. Vitaly, M. Marklund and M. Modestov, "The Rayleigh-Taylor Instability and Internal Waves in Quantum Plasmas,” Physics Letters A, Vol. 372, No. 17, 2008, pp. 3042-3045. doi:10.1016/j.physleta.2007.12.065

[29] J. T. Cao, H. J. Ren, Z. W. Wu and P. K. Chu, “Quantum Effects on Rayleigh-Taylor Instability in Magnetized Plasma,” Physics of Plasmas, Vol. 15, 2008, p. 012110. doi:10.1063/1.2833588

[30] G. A. Hoshoudy, “Quantum Effects on Rayleigh-Taylor Instability in a Vertical Inhomogeneous Rotating Plasma,” Physics of Plasmas, Vol. 16, 2009, p. 024501. doi:10.1063/1.3080202

[31] G. A. Hoshoudy, “Quantum Effects on Rayleigh-Taylor Instability in a Horizontal Inhomogeneous Rotating Plasma,” Physics of Plasmas, Vol.16, 2009, p. 064501. doi:10.063/1.3140038

[32] M. Modestov, V. Bychkov and M. Marklund, "The Rayleigh-Taylor Instability in Quantum Magnetized Plasma with Para- and Ferromagnetic Properties,” Physics of Plasmas, Vol. 16, 2009, p. 032106. doi:10.1063/1.3085796

[33] G. A. Hoshoudy, "Quantum Effects on the RayleighTaylor Instability of Stratified Fluid/Plasma through Porous Media,” Physics Letters A, Vol. 373, No. 30, 2009, pp. 2560-2567. doi:10.1016/j.physleta.2009.05.036

[34] S. Ali, Z. Ahmed, M. Arshad Mirza and I. Ahmad, "Rayleigh-Taylor/Gravitational Instability in Dense Magnetoplasmas,” Physics Letters A, Vol. 373, 2009, pp. 2940-2946. doi:10.1016/j.physleta.2009.06.021

[35] L. C. Gardner, "The Quantum Hydrodynamic Model for Semiconductor Devices," SIAM Journal on Applied Mathematics, Vol. 54, No. 2, 1994, pp. 409-427. doi:10.1137/S0036139992240425 


\section{Appendix}

Here, if we put

$$
\begin{aligned}
& \boldsymbol{U}_{1}=\left(u_{x 1}, u_{y 1}, u_{z 1}\right), \boldsymbol{g}=(0,0,-g), \boldsymbol{Q}_{1}=\left(Q_{x 1}, Q_{y 1}, Q_{z 1}\right) \\
& \frac{\rho_{0}}{\varepsilon}\left\{1+\lambda \frac{\partial}{\partial t}\right\} \frac{\partial u_{x 1}}{\partial t}=\left\{1+\lambda \frac{\partial}{\partial t}\right\}\left[-\frac{\partial p}{\partial t}+Q_{x 1}\right]-\frac{\rho_{0}}{\varepsilon}\left\{1+\lambda_{0} \frac{\partial}{\partial t}\right\}\left[\frac{\varepsilon v_{0}}{k_{1}} u_{x 1}\right] \\
& \frac{\rho_{0}}{\varepsilon}\left\{1+\lambda \frac{\partial}{\partial t}\right\} \frac{\partial u_{y 1}}{\partial t}=\left\{1+\lambda \frac{\partial}{\partial t}\right\}\left[-\frac{\partial p}{\partial y}+Q_{y 1}\right]-\frac{\rho_{0}}{\varepsilon}\left\{1+\lambda_{0} \frac{\partial}{\partial t}\right\}\left[\frac{\varepsilon v_{0}}{k_{1}} u_{y 1}\right] \\
& \frac{\rho_{0}}{\varepsilon}\left\{1+\lambda \frac{\partial}{\partial t}\right\} \frac{\partial u_{z 1}}{\partial t}=\left\{1+\lambda \frac{\partial}{\partial t}\right\}\left[-\frac{\partial p}{\partial z}-\rho_{1} g+Q_{z 1}\right]-\frac{\rho_{0}}{\varepsilon}\left\{1+\lambda_{0} \frac{\partial}{\partial t}\right\}\left[\frac{\varepsilon v_{0}}{k_{1}} u_{z 1}\right] \\
& \frac{\partial \rho_{1}}{\partial t}+\frac{1}{\varepsilon} u_{z 1} \frac{\mathrm{d} \rho_{0}(z)}{\partial z}=0 \\
& \frac{\partial u_{x 1}}{\partial x}+\frac{\partial u_{y 1}}{\partial y}+\frac{\partial u_{z 1}}{\partial z}=0 \\
& Q_{x 1}=\frac{\hbar^{2}}{2 m_{e} m_{i}} \frac{\partial}{\partial x}\left\{\frac{1}{2} \frac{\mathrm{d}^{2} \rho_{1}}{\mathrm{~d} z^{2}}-\frac{1}{2 \rho_{0}} \frac{\mathrm{d} \rho_{0}}{\mathrm{~d} z} \frac{\mathrm{d} \rho_{1}}{\mathrm{~d} z}+\left\{\frac{1}{2}\left(\frac{\partial^{2}}{\partial x^{2}}+\frac{\partial^{2}}{\partial y^{2}}\right)-\frac{1}{2 \rho_{0}} \frac{\mathrm{d}^{2} \rho_{0}}{\mathrm{~d} z^{2}} \frac{1}{2 \rho_{0}^{2}}\left(\frac{\mathrm{d} \rho_{0}}{\mathrm{~d} z}\right)^{2}\right\} \rho_{1}\right\} \\
& Q_{y 1}=\frac{\hbar^{2}}{2 m_{e} m_{i}} \frac{\partial}{\partial y}\left\{\frac{1}{2} \frac{\mathrm{d}^{2} \rho_{1}}{\mathrm{~d} z^{2}}-\frac{1}{2 \rho_{0}} \frac{\mathrm{d} \rho_{0}}{\mathrm{~d} z} \frac{\mathrm{d} \rho_{1}}{\mathrm{~d} z}+\left\{\frac{1}{2}\left(\frac{\partial^{2}}{\partial x^{2}}+\frac{\partial^{2}}{\partial y^{2}}\right)-\frac{1}{2 \rho_{0}} \frac{\mathrm{d}^{2} \rho_{0}}{\mathrm{~d} z^{2}} \frac{1}{2 \rho_{0}^{2}}\left(\frac{\mathrm{d} \rho_{0}}{\mathrm{~d} z}\right)^{2}\right\} \rho_{1}\right\} \\
& Q_{z 1}=\frac{\hbar^{2}}{2 m_{e} m_{i}} \cdot\left\{\frac{1}{2} \frac{\mathrm{d}^{3} \rho_{1}}{\mathrm{dz} z^{3}}-\frac{1}{\rho_{0}} \frac{\mathrm{d} \rho_{0}}{\mathrm{~d} z} \frac{\mathrm{d}^{2} \rho_{1}}{\mathrm{dz} z^{2}}+\left\{\frac{1}{2}\left(\frac{\partial^{2}}{\partial x^{2}}+\frac{\partial^{2}}{\partial y^{2}}\right)-\frac{1}{\rho_{0}} \frac{\mathrm{d}^{2} \rho_{0}}{\mathrm{~d} z^{2}}+\frac{3}{2 \rho_{0}^{2}}\left(\frac{\mathrm{d} \rho_{0}}{\mathrm{~d} z}\right)^{3}\right\} \frac{\mathrm{d} \rho_{1}}{\mathrm{~d} z}\right. \\
& \left.+\left(-\frac{1}{2 \rho_{0}} \frac{\mathrm{d} \rho_{0}}{\mathrm{~d} z}\left(\frac{\partial^{2}}{\partial x^{2}}+\frac{\partial^{2}}{\partial y^{2}}\right)+\frac{1}{2 \rho_{0}^{2}} \frac{\mathrm{d} \rho_{0}}{\mathrm{~d} z} \frac{\mathrm{d}^{2} \rho_{0}}{\mathrm{~d} z^{2}}-\frac{1}{\rho_{0}^{3}}\left(\frac{\mathrm{d} \rho_{0}}{\mathrm{~d} z}\right)^{3}\right) \rho_{1}\right\}
\end{aligned}
$$

Now, Using the expression (10) in the system of equations (30)-(37) maybe written as

$$
\begin{aligned}
& \frac{\rho_{0}}{\varepsilon}\left\{(-i \omega)+\frac{\varepsilon v_{0}}{k_{1}} \frac{1-\lambda_{0}(i \omega)}{1-\lambda(i \omega)}\right\} u_{x 1}=-i k_{x} p_{1}+\bar{Q}_{x 1} \\
& \frac{\rho_{0}}{\varepsilon}\left\{(-i \omega)+\frac{\varepsilon v_{0}}{k_{1}} \frac{1-\lambda_{0}(i \omega)}{1-\lambda(i \omega)}\right\} u_{y 1}=-i k_{y} p_{1}+\bar{Q}_{y 1}
\end{aligned}
$$

$$
\frac{\rho_{0}}{\varepsilon}\left\{(-i \omega)+\frac{\varepsilon v_{0}}{k_{1}} \frac{1-\lambda_{0}(i \omega)}{1-\lambda(i \omega)}\right\} u_{z 1}=-\frac{\partial p_{1}}{\partial z}-\rho_{1} g+\bar{Q}_{z 1}
$$

$$
\bar{Q}_{x 1}=\frac{\hbar^{2} k_{x}}{2 \varepsilon \omega m_{e} m_{i}}\left\{\frac{1}{2} \frac{\mathrm{d} \rho_{0}}{\mathrm{~d} z} \frac{\mathrm{d}^{2} u_{z 1}}{\mathrm{~d} z^{2}}+\left(\frac{\mathrm{d}^{2} \rho_{0}}{\mathrm{~d} z^{2}}-\frac{1}{2 \rho_{0}}\left(\frac{\mathrm{d}^{2} \rho_{0}}{\mathrm{~d} z^{2}}\right)^{2}\right) \frac{\mathrm{d} u_{z 1}}{\mathrm{~d} z}+\left(\frac{1}{2} \frac{\mathrm{d}^{3} \rho_{0}}{\mathrm{dz} z^{3}}-\frac{1}{\rho_{0}} \frac{\mathrm{d} \rho_{0}}{\mathrm{~d} z} \frac{\mathrm{d}^{2} \rho_{0}}{\mathrm{~d} z^{2}}-\frac{k^{2}}{2} \frac{\mathrm{d} \rho_{0}}{\mathrm{~d} z}+\frac{1}{2 \rho_{0}^{2}}\left(\frac{\mathrm{d} \rho_{0}}{\mathrm{~d} z}\right)^{3}\right) u_{z 1}\right\}
$$

$$
\bar{Q}_{y 1}=\frac{k_{y}}{k_{x}} \bar{Q}_{x 1}
$$




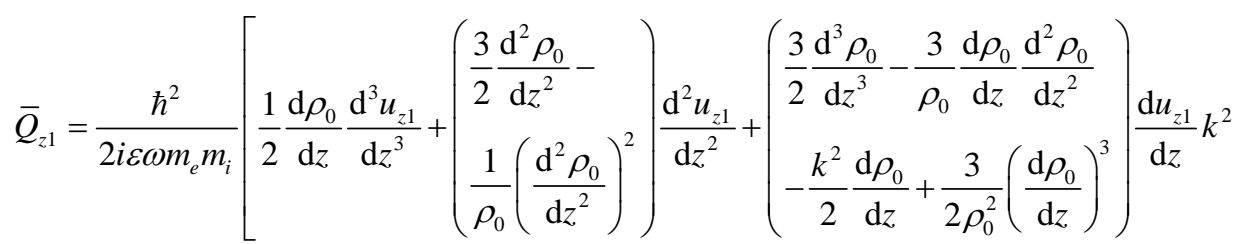

$$
\begin{aligned}
& +\left(\frac{1}{2} \frac{\mathrm{d}^{4} \rho_{0}}{\mathrm{~d} z^{4}}-\frac{1}{\rho_{0}} \frac{\mathrm{d} \rho_{0}}{\mathrm{~d} z} \frac{\mathrm{d}^{3} \rho_{0}}{\mathrm{~d} z^{3}}-\frac{k^{2}}{2} \frac{\mathrm{d}^{2} \rho_{0}}{\mathrm{~d} z^{2}}-\frac{1}{\rho_{0}}\left(\frac{\mathrm{d}^{2} \rho_{0}}{\mathrm{~d} z^{2}}\right)^{2}+\frac{5}{2 \rho_{0}^{2}}\left(\frac{\mathrm{d} \rho_{0}}{\mathrm{~d} z}\right)^{2} \frac{\mathrm{d}^{2} \rho_{0}}{\mathrm{~d} z^{2}}+\frac{k^{2}}{2 \rho_{0}}\left(\frac{d \rho_{0}}{d z}\right)^{2}-\frac{1}{\rho_{0}^{3}}\left(\frac{\mathrm{d} \rho_{0}}{\mathrm{~d} z}\right)^{4}\right)
\end{aligned}
$$

